\title{
Additional Muscle Slip of Bicipital Aponeurosis and its Anomalous Relationship with the Median Cubital Vein
}

\author{
Nandini Bhat, ${ }^{1}$ Kumar M. R. Bhat, ${ }^{2}$ Antony S. D'Souza, ${ }^{1}$ Sushma R. Kotian ${ }^{1}$
}

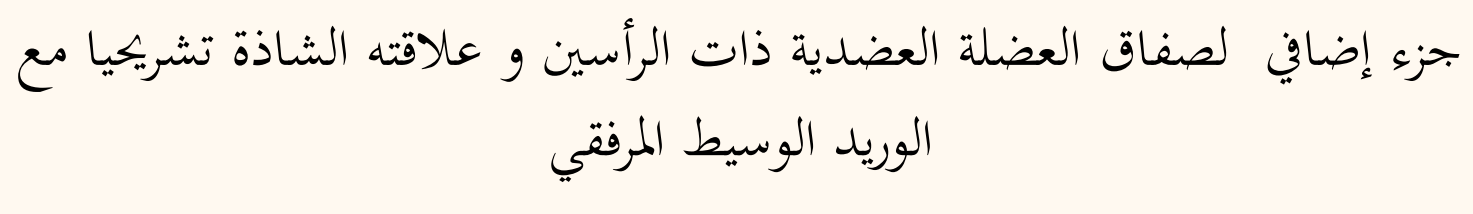

نانديني بهات، كومار بهات، أنتوني سيلفانديسوزا، سوشما كوتيان

ABSTRACT: The cubital region of the arm is a common site for recording blood pressure, taking blood for analysis and administering intravenous therapy and blood transfusions. During the routine dissection of a 70-year-old male cadaver at the Kasturba Medical College, Manipal, Karnataka, India, in 2015, it was observed that the aponeurotic insertion of the biceps brachii muscle divided into two slips. The medial slip fused normally with the deep fascia of the forearm, while flexor carpi radialis muscle fibres originated from the lateral slip. There was also a single vein in the forearm, the cephalic vein, which bifurcated to form the median cubital vein and the cephalic vein proper. The median cubital vein, further reinforced by the radial vein, passed deep to the two slips of the bicipital aponeurosis and then continued as the basilic vein. During venepuncture, medical practitioners should be aware of potential cubital fossa variations which could lead to nerve entrapment syndromes.

Keywords: Anatomic Variation; Elbow; Aponeurosis, abnormalities; Case Report; India.

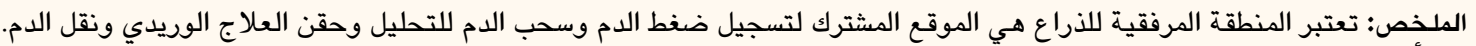

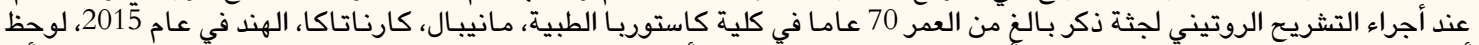

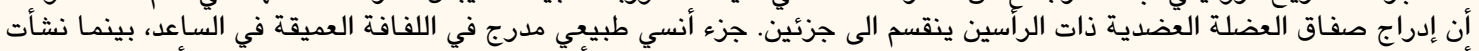

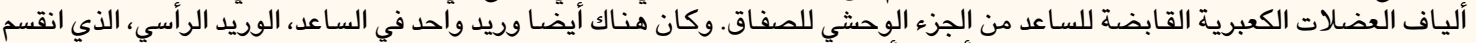

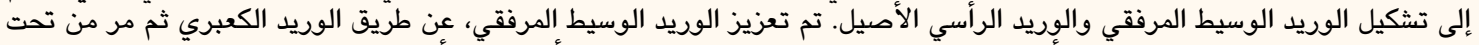

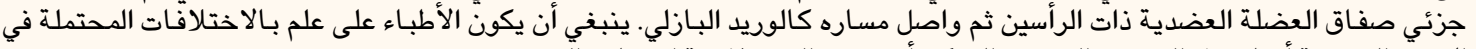

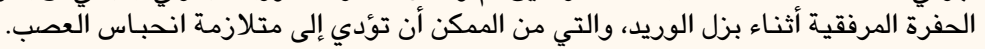

الكلمات المفتاحية: التغير التشريحي؛ كوع؛ صفاق، تشوهـات؛ تقرير الحالة؛ الهند.

T he cubital fossa is a Depression on the anterior aspect of the elbow containing the median nerve, the brachial artery, the tendon of the biceps brachii (BB) and the radial nerve; the roof of the cubital fossa is formed by the continuity of brachial and antebrachial fascia and reinforced by bicipital aponeurosis (BA), subcutaneous tissue and skin. ${ }^{1}$ The BB forms a flattened tendon at the elbow joint and then inserts the radial tuberosity; close to the insertion point, the tendon provides an expansion which is broad and extends medially (i.e. the BA). The BA passes across the brachial artery and deep to the median cubital vein (MCV) and fuses with the deep fascia of the forearm. ${ }^{1}$ Due to its insertion away from the tendon of the $\mathrm{BB}$, the $\mathrm{BA}$ disperses some of the power in a different direction and thus helps in the dual action of the BB as both a supinator and flexor muscle of the forearm. ${ }^{2}$ The BA reinforces the fascia of the cubital fossa and protects the underlying median nerve and brachial artery, which is important during venepuncture of the $\mathrm{MCV}{ }^{1}$

The muscles of the superficial flexor compartment of the forearm-the pronator teres (PT), flexor carpi radialis (FCR), palmaris longus, flexor digitorum superficialis and flexor carpi ulnaris muscles-arise from the medial epicondyle of the humerus by a common tendon. These muscles have an additional attachment to the antebrachial fascia near the elbow and the septa that passes from this fascia between individual muscles. ${ }^{1}$ However, these muscles may sometimes arise from the BA; such variable attachments to the BA can lead to an entrapment syndrome of the median nerve or ischaemic contracture due to compression of the brachial artery. ${ }^{2,3}$

The BA normally forms a support for the overlying $\mathrm{MCV}$ and aids in its identification and palpation for diagnostic purposes. ${ }^{2}$ The MCV connects the cephalic and basilic veins distal to the elbow and lies in the 


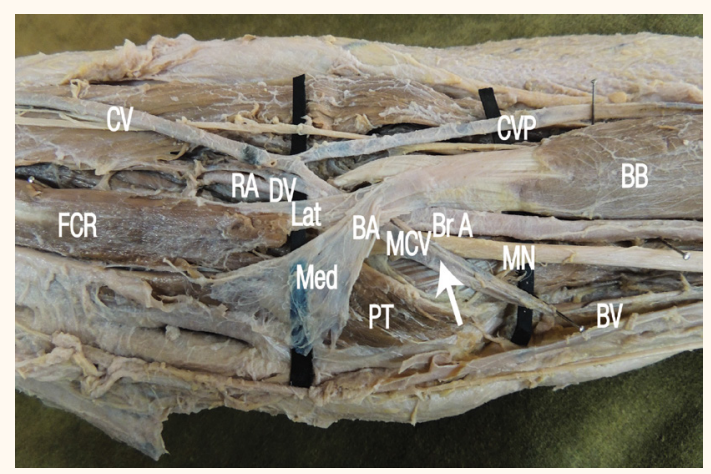

Figure 1: Photograph of the dissected right cubital fossa of a 70-year-old male cadaver showing an additional muscle slip from the bicipital aponeurosis (BA) to the flexor carpi radialis. The median cubital vein can be observed located deep to the BA (arrow).

$C V=$ cephalic vein $; C V P=$ cephalic vein proper $; R A=$ radial artery; $D V=$ deep vein accompanying the $R A ; B B=$ biceps brachii Lat = lateral BA slip; $B A=$ bicipital aponeurosis; $B r A=$ brachial artery; $F C R=$ flexor carpi radialis; $M C V=$ median cubital vein; $M N=$ median nerve; Med = medial BA slip; $P T=$ pronator teres; $B V=$ basilic vein

superficial fascia of the cubital fossa, which is always superficial to the BA. ${ }^{1}$ Many adverse effects can occur following venous mispuncture, such as bruising, haematomas and sensory changes. ${ }^{4}$ In clinical practice, knowledge of the anatomy of the MCV is important as this vein is frequently used for venepuncture during blood withdrawal and transfusion procedures, cardiac catheterisation, insertion of peripheral vascular grafts and for the creation of arteriovenous fistulae. ${ }^{5}$ Knowledge of any variations in the cubital region is therefore helpful to avoid unwanted complications during routine cubital fossa procedures.

\section{Case Report}

A routine undergraduate dissection session of the right cubital fossa of a 70-year-old male cadaver was undertaken at the Kasturba Medical College, Manipal, Karnataka, India, in 2015. It was observed that the $\mathrm{BA}$ arose from the tendon of the $\mathrm{BB}$, just proximal to the intercondylar line of the humerus. This aponeurosis then divided to form a broad thin medial slip and a thick slender lateral slip. The medial slip was superficial to the cubital structures and merged with the deep fascia of the forearm, while the lateral slip passed vertically downwards towards the forearm, medial to the tendon of the BB. In the forearm, this thick extension of the bicipital tendon provided an additional origin to the muscular slips of the FCR. These fleshy muscular fibres then fused with the rest of the FCR muscle, which originated from the medial epicondyle along with the other flexor muscles of the forearm.
No abnormalities were observed in the dorsal venous network. The cephalic vein was the only vein present in the lateral side of the forearm and it continued as a single vein. There was a complete absence of the basilic vein. Instead, many smaller veins from the medial side of the forearm joined the cephalic vein. As the cephalic vein reached the cubital fossa, it bifurcated with the lateral branch, continuing into the arm as the cephalic vein proper while the medial branch passed obliquely under both BA slips towards the medial side of the arm. In the cubital region, the medial branch (i.e. the $\mathrm{MCV}$ ) was reinforced by a deep vein accompanying the radial artery and continued as the basilic vein in the medial side of the arm [Figure 1].

\section{Discussion}

In humans, the muscles of the upper limbs develop from the mesenchyme of the paraxial mesoderm during the fifth week of fetal development. ${ }^{6}$ Myoblasts are stimulated to migrate into the developing limb buds by several growth factors produced by cells in the proximal limb bud. These pre-muscle cells express adhesion molecules that are essential to their adequate distribution throughout the limb. ${ }^{7}$ The presence of additional slips from the BA and the variant origin of the FCR from the BA may indicate changes in the formation and structure of the myotome of a somite or in the distribution of cell adhesion molecules on pre-muscle cells. ${ }^{6,7}$

Several anomalous anatomic variations of the cubital region of the arm have been previously described in the literature. Bhat et al. reported an additional muscular slip from the BA to the FCR and PT. ${ }^{8}$ Similarly, Trivedi et al. described a case wherein additional muscle slips originated from the belly of the $\mathrm{BB}$ and were connected to the FCR and PT. ${ }^{9}$ However, in the present case, only fleshy FCR muscle fibres originated from the lateral slip of the BA, without any accompanying PT fibres. Nayak et al. reported a case of BA bifurcation into a larger medial slip and a small lateral slip. ${ }^{3}$ The medial slip, instead of fusing with the deep fascia on the medial side of the forearm, merged with the common flexor muscles. A few fleshy fibres of the PT and FCR arose from this slip and the lateral slip merged with the brachioradialis muscle. ${ }^{3}$ Deopujari et al. reported three BA variants, including thickened tendinous slips bordering the aponeurosis, a third head of the BB muscle forming the aponeurosis and the aponeurosis giving rise to some muscle fibres joining the FCR muscle. ${ }^{10}$ 
Paval et al. described a case with a BB with a normal origin and course, but with a variant insertion. ${ }^{11}$ At the insertion, most of the fibres were inserted as a rounded tendon into the radial tuberosity; however, a few fibres from the medial side formed a tendinous slip which further bifurcated, with the brachial artery and median nerve passing between the two slips. The lateral slip merged with the fascia covering the flexor carpi ulnaris muscle, while the medial slip inserted into the medial supracondylar ridge. ${ }^{11}$ Such variations of the BA may lead to neurovascular symptoms due to entrapment of the underlying vessels and nerves. ${ }^{2,3}$ Extra slips from the BA may function independently and result in stress concentration on the bony areas of the attachment. ${ }^{11}$

Variations are common in the superficial veins of the upper limb. Jain et al. reported the absence of the MCV with the existence of an accessory cephalic vein. ${ }^{12}$ In a study of variations of the superficial veins of the upper limbs of 169 living Caucasian individuals, nine patterns of venous drainage were observed..$^{13}$ In another study of the cubital fossa venous arrangements of 135 living Nigerian adults, the most common pattern was the separation of the median antebrachial vein into the median cephalic and median basilic veins which connect to the cephalic and basilic veins. ${ }^{5}$ In the present case, only the cephalic vein was present in the forearm while the basilic vein was absent; the former then bifurcated into the cephalic vein proper and the MCV, which subsequently continued as the basilic vein. Using a venous illuminator, Lee et al. classified the pattern of superficial veins in the cubital fossa of 200 Korean patients into four types according to the presence of the MCV; in all cases, the MCV was superficial in the cubital fossa. ${ }^{14}$

Previous research has described variations in the superficial veins of the upper limbs in relation to nearby structures and also the absence of some of the usual veins., ${ }^{5,14,15}$ Interestingly, in the present case, the MCV was located deep to both BA slips. This finding is unusual and, to the best of the author's knowledge, has not yet been reported in the literature. Routine venepuncture procedures in individuals with deeply located MCVs would be difficult and painful. Therefore, awareness of the possible existence of such a variation is essential when carrying out procedures involving the MCV in the cubital fossa. In these cases, the use of a venous illuminator during venepuncture procedures may be beneficial. ${ }^{14,15}$

\section{Conclusion}

The present case report described a rare anomaly in which the FCR originated from the BA and the MCV lay deep to the aponeurosis. These findings may be helpful for medical practitioners to encourage awareness of potential variations in the cubital region which may have an impact on diagnostic and therapeutic procedures.

\section{References}

1. Standring S. Gray's Anatomy: The anatomical basis of clinical practice, 40th ed. London, UK: Churchill Livingstone-Elsevier, 2008. Pp. 825-6.

2. Joshi SD, Yogesh AS, Mittal PS, Joshi SS. Morphology of the bicipital aponeurosis: A cadaveric study. Folia Morphol (Warsz) 2014; 73:79-83. doi: 10.5603/FM.2014.0011.

3. Nayak SB, Swamy RS, Shetty P, Maloor PA, Dsouza MR. Bifurcated bicipital aponeurosis giving origin to flexor and extensor muscles of the forearm: A case report. J Clin Diagn Res 2016; 10:AD01-02. doi: 10.7860/JCDR/2016/17714.7215.

4. Newman BH, Pichette S, Pichette D, Dzaka E. Adverse effects in blood donors after whole-blood donation: A study of 1000 blood donors interviewed 3 weeks after whole-blood donation. Transfusion 2003; 43:598-603. doi: 10.1046/j.15372995.2003.00368.x.

5. Ukoha UU, Oranusi CK, Okafor JI, Ogugua PC, Obiaduo AO. Patterns of superficial venous arrangement in the cubital fossa of adult Nigerians. Niger J Clin Pract 2013; 16:104-9. doi: 10.41 03/1119-3077.106777.

6. Larsen WJ. Human Embryology, 2nd ed. Philadelphia, Pennsylvania, USA: Saunders, 1997. Pp. 311-39.

7. Carlson BM. Human Embryology and Developmental Biology, 3rd ed. Maryland Heights, Missouri, USA: Mosby, 2004. Pp. 224-5.

8. Bhat KM, Kulakarni V, Gupta C. Additional muscle slips from the bicipital aponeurosis and a long communicating branch between the musculocutaneous and the median nerves. Int J Anat Var 2012; 5:41-3.

9. Trivedi S, Sinha MB, Sharma DK, Rathore M, Siddiqui AU. Abnormal musculotendinous slip from biceps brachii to pronator teres: A case report. Int J Biomed Adv Res 2015; 6:64-6. doi: 10.7469/ijbar.v6i1.1527.

10. Deopujari R, Quadir N, Athavale S, Gajbhiye V, Kotgirwar S. Variant bicipital aponeurosis: A cadaveric study. Peoples J Sci Res 2014; 7:43-6.

11. Paval J, Mathew JG. A rare variation of the biceps brachi muscle. Indian J Plast Surg 2006; 39:65-7. doi: 10.4103/09700358.26907.

12. Jain T, Yadav SK. Case study: Variation of superficial veins pattern of upper limb found in dissection. Int Ayurvedic Med J 2015; 3:2223-5.

13. Vasudha TK. A study on superficial veins of upper limb. Natl J Clin Anat 2013; 2:204-8.

14. Lee H, Lee SH, Kim SJ, Choi WI, Lee JH, Choi IJ. Variations of the cubital superficial vein investigated by using the intravenous illuminator. Anat Cell Biol 2015; 48:62-5. doi: 10.51 15/acb.2015.48.1.62.

15. Yamada K, Yamada K, Katsuda I, Hida T. Cubital fossa venipuncture sites based on anatomical variations and relationships of cutaneous veins and nerves. Clin Anat 2008; 21:307-13. doi: 10.1002/ca.20622. 